\title{
Radiologic Screening for Breast Cancer: Current Controversies
}

\author{
Carol H. Lee
}

Published online: 20 December 2013

(c) Springer Science+Business Media New York 2013

\begin{abstract}
Breast cancer is the most commonly occurring cancer, aside from skin cancer, among American women and the second leading cause of cancer death. Screening with mammography has been used for decades in this country, and since its introduction, there has been a reduction in breast cancer mortality. However, controversy surrounding the use of mammography to screen for breast cancer continues. In addition, the development of newer imaging techniques that can be applied to breast cancer screening has generated further debate about the value and appropriate use of radiologic imaging for breast cancer screening.
\end{abstract}

Keywords Screening · Breast cancer - Mammography · Tomosynthesis - Breast magnetic resonance imaging · Breast ultrasound

\section{Introduction}

Several prospective randomized trials conducted in the US and Europe in the 1970s and 1980s showed statistically significant breast cancer mortality reduction associated with mammographic screening [1-3]. Based on these results, a number of medical organizations in the US have advocated its use. Throughout its history, however, mammographic screening has been the subject of debate, and

This article is part of the Topical Collection on Essentials in Oncologic Imaging.

C. H. Lee $(\bowtie)$

Memorial Sloan-Kettering Cancer Center, 300 East 66th Street, New York, NY 10065, USA

e-mail: leec1@mskcc.org despite the randomized trials and 30 years of experience, controversy surrounding screening mammography has intensified rather than diminished and polarization between advocates and critics of screening has increased. Proponents of screening mammography point out the decrease in breast cancer mortality, which they say can be directly attributed to screening while opponents state that treatment rather than early detection is responsible for the observed mortality reduction, and there is little justification for mass screening with mammography.

Other areas of controversy include at what age screening mammography should start, how often it should occur and whether the harms of screening, which include false-positive results and overdiagnosis, outweigh the benefits. Finally, increased awareness of the limitations of mammography and the development of other imaging techniques to screen for breast cancer, such as tomosynthesis, ultrasound, and magnetic resonance imaging (MRI), among others, have raised new questions about what modality should be used for which population of women.

\section{Controversies Surrounding Screening Mammography}

Doubts About Efficacy

Meta-analyses of the randomized controlled trials of screening mammography show a statistically significant 15-22\% reduction in breast cancer mortality among the population invited to screening $[4,5]$. Despite this, there are continued challenges to the validity of screening mammography. Goetzche and Olsen in 2000 reviewed the screening trials and declared that all but three had serious flaws in methodology and poor randomization rendering their results unreliable [6]. The three that they deemed to 
have proper methodology showed no reduction in breast cancer mortality among the screened population, showing a relative risk of 1.04 (95\% CI 0.94-1.05). Therefore, they stated, screening with mammography is unjustified. Other studies of the efficacy of screening comparing mortality rates in screened groups with those in either historical or geographical controls have yielded varying results despite being based on data that include the same population and same time periods.

In a report from Denmark, mortality rates among screened and unscreened populations were compared and showed a $1 \%$ decline among the screened group but a $2 \%$ decline among the unscreened group [7]. In addition, the greatest decrease in mortality from breast cancer occurred in women who were too young to be eligible for screening. The authors of this study postulated that the decrease in breast cancer mortality was due to improvements in therapy and greater awareness of breast cancer rather than early detection through screening. Similarly, a study of breast cancer mortality trends in four counties in Sweden from 1972 to 2009 found that in two counties, breast cancer mortality trends after screening was introduced was similar to those before the use of screening [8]. The authors concluded that screening mammography had little or no effect on breast cancer mortality.

Finally, a study of screening in Norway compared death rates in regions with and without screening to historical data from the same areas and found that deaths declined by 7.2 deaths/100,000 person-years in the screening group compared to a decline of $4.8 / 100,000$ person-years in the non-screened group [9]. The conclusion of this study was that screening accounted for only a third of the observed mortality reduction.

Countering these studies are others that continue to demonstrate a benefit of screening. A meta-analysis of the randomized controlled trials by an independent panel in the UK (UK) reported a $20 \%$ mortality reduction among screened populations [10॰•]. An analysis of data from the two-county Swedish trial after 29 years of follow-up reported a statistically significant decline in mortality of $31 \%$ in the screening group [11]. A recent case-control study from Australia reported a $49 \%$ reduction in breast cancer mortality among women receiving screening [12]. In addition, researchers conducted an analysis of 7,301 women with breast cancer in Massachusetts, 609 of whom died of the disease [13•]. They found that $71 \%$ of the deaths occurred in women who had either never been screened or who had not had screening in the past 2 years and concluded that screening is associated with a lower risk of dying from breast cancer.

All of these reports, both in favor of and opposed to screening, are met with criticisms of methodology, underlying assumptions, type of analysis and conclusions by the opposing group. Many of the studies rely on very sophisticated statistical methods that are difficult if not impossible for a non-statistician or epidemiologist to decipher. This has left clinicians and the general public confused as to where the truth lies.

\section{When Should Screening Start?}

The age at which regular screening mammography should begin has been a point of contention for many years and is still being debated. This topic was hotly contested in the 1990s, and the argument was re-ignited in 2009 when the US Preventive Services Task Force (USPSTF) revised their previous guidelines to suggest that routine screening should wait until age 50 [14]. They and others cite the lower incidence of breast cancer, the increased proportion of false positives, and the lower sensitivity of mammography among younger women as justification for their recommendation.

Evidence of benefit for women aged 40-49 from the randomized controlled trials was limited, as most were not specifically designed to address this question. A trial that did look at this was conducted in Canada and showed no significant difference in mortality between the screened and unscreened population [15]. In addition, the AGE trial conducted in the UK randomly assigned women aged 39-41 to screening or no screening and after 11 years of follow-up showed a nonsignificant $11 \%$ reduction in mortality among the screening group [16]. The USPSTF performed a meta-analysis of eight screening trials involving women aged 39-49 and found a significant $15 \%$ reduction in mortality [5]. They calculated that the number needed to be screened to save one life among the 40-49year age group was 1,904 compared to 1,339 among 50-59 year olds and 337 among those aged 60-69. They also found that starting at age 40 resulted in the greatest number of years of life saved. However, citing the number of false positives generated by screening among the younger age group, the USPSF recommended that routine screening start at age 50, and for women in their 40s the decision as to whether or not to screen should be an individual one depending on risk factors and values concerning possible risks versus benefits. In contrast, the American Cancer Society (ACS), American Congress of Obstetrics and Gynecology (ACOG), American College of Radiology (ACR) and the American College of Surgeons (ACoS), in recognition of the benefits of screening in the younger age group, recommend that routine screening begin at age 40 . Guidelines in many European countries call for screening of average risk women to begin at age 50. A summary of screening mammography recommendations from a number of organizations in the US and from national screening programs around the world is presented in Table 1. 
Table 1 Screening mammography recommendations for average risk women

\begin{tabular}{|c|c|c|c|}
\hline Country/organization & $\begin{array}{l}\text { Age to start routine } \\
\text { screening }\end{array}$ & $\begin{array}{l}\text { Age to stop } \\
\text { screening }\end{array}$ & Interval \\
\hline $\begin{array}{l}\text { United States/ACS, } \\
\text { ACR, ACOG, ACoS }\end{array}$ & 40 & $\begin{array}{l}\text { As long as a woman } \\
\text { is in good health }\end{array}$ & Yearly \\
\hline USPSTF & 50 & 74 & Every 2 years \\
\hline Canada & 50 & 74 & Every $2-3$ years \\
\hline United Kingdom & 47 & 73 & Every 3 years \\
\hline European Union ${ }^{\mathrm{a}}$ & 50 & 69 & Every 2 or 3 years \\
\hline Sweden & 40 & 74 & $\begin{array}{l}\text { Every } 1.5 \text { years } 40-49 \\
\text { Every } 2 \text { years } \geq 50\end{array}$ \\
\hline Australia & 50 & 74 & Every 2 years \\
\hline
\end{tabular}

ACS American Cancer Society, ACR American College of Radiology, ACOG American Congress of Obstetrics and Gynecology, ACoS American College of Surgeons, USPSTF US Preventive Services Task Force

${ }^{a}$ Varies by country, most common strategy listed here

\section{How Often Should Screening Be Performed?}

The interval at which screening mammography should be performed is another area of debate. Using six different models and data from the Breast Cancer Surveillance Consortium (BCSC), Mandelblatt et al. [17] weighed the benefits of various screening models against the risks of false-positive exams, subsequent benign biopsies and overdiagnosis. All of the models indicated that the most efficient strategy was for biennial rather than annual screening. Also using data from the BCSC, Kerlikowski et al. [18] evaluated tumor stage, size and lymph node status as a function of age, breast density and screening interval. They found that biennial screening was not significantly associated with adverse tumor characteristics in most women except those aged 40-49 with extremely dense breasts in whom biennial compared to annual screening was associated higher stage disease. In the US, the ACS, ACOG, ACR and ACoS recommend annual screening, whereas the USPSTF recommends biennial screening. In most of Europe, the recommended interval is 2 years, and in the UK it is 3 years (Table 1).

\section{Do the Benefits of Screening Mammography Outweigh} the Harms?

The benefit of screening is the potential of avoiding death from breast cancer through early detection. Some of the harms such as discomfort from the exam, radiation exposure and psychological distress from an abnormal interpretation, though important, are not generally regarded as being of sufficient magnitude to outweigh the benefit. Other risks, however, namely false positives necessitating additional imaging and/or biopsy and most notably the risk of overdiagnosis, have generated a great deal of controversy in terms of the balance of harms versus benefit for screening mammography.

\section{False Positives}

As with any screening test, mammography can result in an abnormal reading that requires further evaluation, usually consisting of additional mammographic views and/or ultrasound. On occasion, a biopsy is necessary to exclude malignancy. The degree to which false-positive interpretations occurs varies from location to location, but in the United States is generally on the order of $10 \%$ [19]. Christiansen et al. [20] estimated the cumulative risk for an abnormal reading to be $43 \%$ after nine rounds of screening. False positives have been cited as a reason for not screening the 40-49 year age group and are also given as a reason to screen biennially rather than annually. In the modeling study by Mandelblatt et al. [17], biennial screening resulted in similar stage distribution of cancers detected, but nearly $50 \%$ reduction in false-positive readings and benign biopsies.

Abnormal findings on screening mammography have been reported to be associated with anxiety, and this has been cited as a "harm" associated with screening [14]. However, the reported degree of psychological distress and the significance of this anxiety varies among different reports [21], and acceptance of false-positive results among the public seems to be high. In one survey of 503 American women, $63 \%$ felt that 500 false positives were reasonable to save one life, and $37 \%$ would tolerate 10,000 or more [22].

\section{Overdiagnosis}

Overdiagnosis of breast cancer is defined as detection of disease that is so biologically innocent that it would never be life-threatening. The possibility of overdiagnosis has been cited as a major harm associated with screening, but 
the degree to which this occurs is difficult to determine, and it ranges from less than $1 \%$ to more than $50 \%[23$, 24]. Recently, Bleyer and Welch [25•], using Surveillance, Epidemiology and End Results data, determined that in the 30 years since screening mammography has been used in the US, the incidence of early stage cancer has doubled, but the incidence of late stage disease has only decreased by $8 \%$. They concluded that this is due to overdiagnosis, which they estimated accounts for $31 \%$ of all breast cancers diagnosed. In contrast, Yen et al. [24] looked at the incidence of cancer in one of the Swedish counties involved in an early randomized controlled screening trial and found no excess incidence among the screened population after 29 years of follow-up. They concluded that overdiagnosis occurred rarely if at all. In addition, a study of screening mammography in Norway estimated that overdiagnosis occurred in $2.3 \%$ of targeted women [26]. In the UK, an independent panel examining existing information on the efficacy of screening concluded that mammography reduces breast cancer mortality by $20 \%$, but is associated with overdiagnosis on the order of $19 \%$ of cancers diagnosed [10•*]. Based on their calculations, the panel postulated that for every breast cancer death avoided through screening, three cases were overdiagnosed. Finally, the USPSTF in their analysis concluded that overdiagnosis likely occurs in $\sim 10 \%$ of cases and is more of a consideration in older women in whom slowly growing cancers may be detected by screening [5].

The discrepancy among published studies in the estimates of overdiagnosis is undoubtedly due to differences in methodology and in underlying assumptions. The crux of the problem of overdiagnosis is the inability to discern the biologic significance of cancers that are found through screening. Rather than addressing overdiagnosis by abandoning screening, as has been suggested by some, development of ways to identify which cancers are potentially lethal and which are not should be the goal.

\section{Other Screening Modalities}

The development of screening modalities other than conventional mammography has provided new opportunities for detection of breast cancer, but also new areas of controversy. Questions raised by other screening modalities include when and on whom they should be used and whether they are cost-effective.

\section{Tomosynthesis}

Digital breast tomosynthesis (DBT) is a digital mammography technique in which multiple low-dose mammographic images are acquired as the X-ray tube moves in an
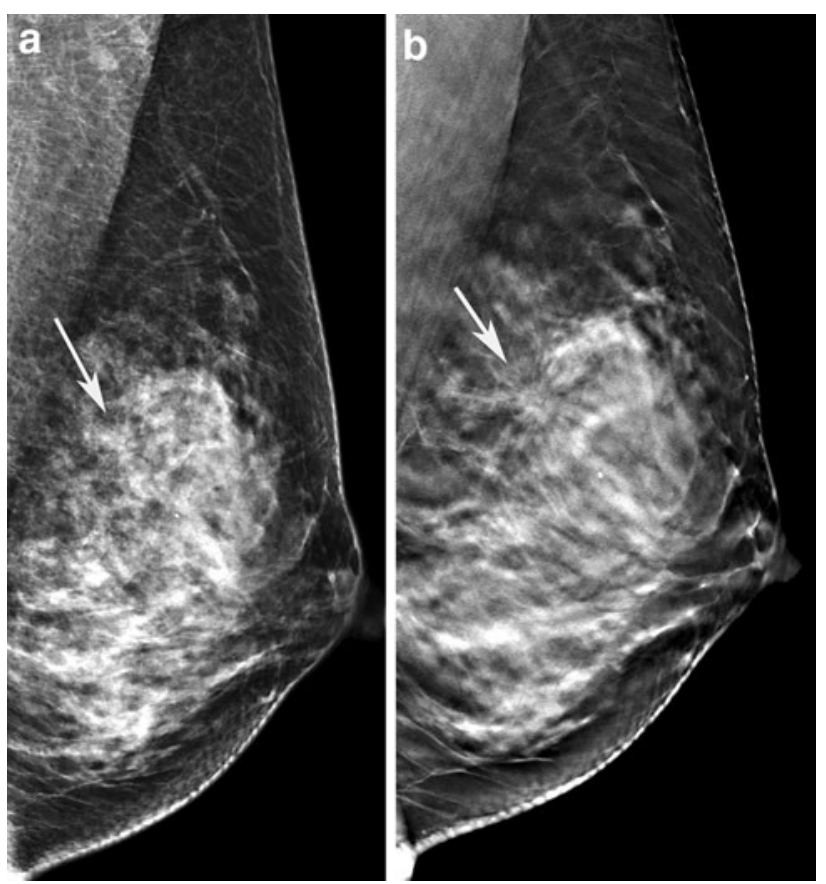

Fig. 1 Example of tomosynthesis image. a Standard 2D left MLO view; b slice from tomosynthesis study obtained at the same time as the standard 2D view. The cancer in the breast is more clearly seen on the DBT image

arc. The images are then processed to create a simulated three-dimensional rendering of the breast that can be displayed as slices, similar to a computed tomography (CT) scan. Unlike CT, however, tomosynthesis images are not truly three-dimensional as one of the axes is computer generated from two-dimensional (2D) data. The theory behind DBT is that by viewing slices of breast tissue and eliminating overlying tissue, cancers might be seen more easily (Fig. 1).

In 2011, the Food and Drug Administration (FDA) approved the first device for clinical use in the US. Approval was granted for DBT to be used only in addition to, not as a substitute for, standard 2D mammography. This was because it could not be demonstrated that the use of DBT alone increased cancer detection over standard 2D mammography alone. The radiation dose from a single DBT view is approximately equal to that of a $2 \mathrm{D}$ view. Therefore, women who have DBT as part of their screening examination receive twice the radiation dose of a $2 \mathrm{D}$ study.

Since its introduction, DBT is increasingly being adopted by practices in the US. Information on its performance is still limited. Several studies have shown that tomosynthesis reduces the number of false-positive interpretations at screening [27-29]. In one of the earliest reports on DBT, Poplack et al. [27] found a $40 \%$ reduction in the recall rate with the use of tomosynthesis in addition to standard mammography. Multi-reader studies conducted 
under the auspices of the equipment manufacturer and published in the peer-reviewed literature reported an increase in sensitivity when DBT was added to 2D mammography and a statistically significant decrease in recall for non-cancer cases among all of the readers [29]. Recall among cancer cases, however, was mixed, with some of the readers showing an increase, others no change, and in some an actual decrease.

Early results of two randomized prospective trials of DBT for screening have recently been published. In one being conducted in Norway, comparison of 12,631 cases read with and without the addition of DBT showed a $27 \%$ increase in the cancer detection rate $(6.1 / 1,000-8 / 1,000)$ accompanied by a $15 \%$ decrease in the recall rate (61.1/ 1,000-53.1/1,000) [30•]. Similarly, a study from Italy showed a statistically significant increase in cancer detection from 5.3/1,000 with 2D mammography to $8.1 / 1,000$ with the addition of DBT. They also showed a $17.2 \%$ reduction in recalls [31].

These results are promising, but two additional reports of DBT as used in routine clinical practice in the US are not as favorable. In one study by Rose et al. [32], the results of screening using 2D plus DBT in 9,499 women were compared to results obtained from screening in 13,856 women prior to the introduction of DBT. There was a significant decrease in recall rate from $8.7-5.5 \%$, but there was no statistically significant change in cancer detection rate. A study by Haas et al. [33] had similar results. In this study, DBT is used with 2D at some but not all sites, and screening results from the sites using DBT were compared to results from those that do not. The recall rate was $12 \%$ at sites not using DBT and $8.4 \%$ at sites that did. This difference was statistically significant. The cancer detection rate, however, was similar with and without DBT at 5.7/1,000 with DBT and 5.2/1,000 without. The final word on how DBT can best be used will be determined by further experience and data on its performance in the clinical setting.

In May 2013, the FDA approved the use of a synthesized 2D mammogram produced from the DBT images as a replacement for the standard $2 \mathrm{D}$ mammogram. This obviates the need for obtaining a separate 2D examination during screening, thus lowering the radiation dose associated with the use of DBT. Whether this synthesized image will be efficacious in replacing the $2 \mathrm{D}$ image remains to be demonstrated, but if it does, one of the major disadvantages of DBT, namely the increased radiation dose, will no longer be a factor.

\section{Ultrasound}

Screening with ultrasound has a number of advantages over other screening modalities. It does not require compression or intravenous contrast and does not deliver ionizing radiation. Ultrasound is widely available and relatively inexpensive, and a number of studies have reported additional cancers detected when screening ultrasound is added to mammography [34-37]. Despite this, the adoption of screening ultrasound has, until recently, been limited in the US largely because of the fact that the study is very operator dependent and relatively time consuming in terms of image acquisition. However, the use of screening breast ultrasound is increasing rapidly in this country because of legislation in a number of states requiring direct patient notification of a woman's breast density after a mammogram. The controversy surrounding screening with ultrasound is not so much whether this modality is effective but whether there should legislation mandating direct patient notification of breast density, which results in increased demand for screening with ultrasound. These laws have been the result of a grassroots movement started by women whose cancers were not detected by mammography because the cancers were obscured by dense tissue [38 • $]$. Currently, 12 states have direct density notification, and another 6 state legislatures are considering similar bills (Fig. 2). In addition, a bill has been introduced in the House of Representatives to make notification a national mandate.

The Breast Imaging Reporting and Data System (BIRADS) of the ACR describes four categories of breast density: almost entirely fat, scattered fibroglandular densities, heterogeneously dense and extremely dense (Fig. 3) [39]. Approximately $10 \%$ of women have predominately fatty breasts, $40 \%$ have scattered fibroglandular tissue, $40 \%$ have heterogeneously dense breasts, and, in about $10 \%$, breast tissue is extremely dense [40]. Breast density is significant in that it is associated with increased risk of breast cancer, and dense breast tissue can obscure cancers, making them harder to detect by mammography. Carney et al. [41] showed sensitivity for screening was $88 \%$ in fatty breasts and $62 \%$ in women with extremely dense breasts. In the Digital Mammographic Imaging Trial that compared film-screen to digital mammography, the sensitivity of film-screen mammography in women with extremely dense breasts was only $55 \%$ [40]. The sensitivity of ultrasound is not decreased by breast density, and with increased awareness among the public of the limitations of mammography, utilization of ultrasound in addition to mammography for screening women with dense breasts has been increasing.

Data from a number of single-center ultrasound screening series and one large multicenter trial have all reported a supplemental yield of screening of $\sim 3 / 1,000$ women screened [34-37]. For all but one of these studies, the ultrasound examinations were radiologist-performed. In three more recent reports of screening ultrasound, all from Connecticut, which was the first state to pass density 


\section{DENSITY NOTIFICATION LAWS}
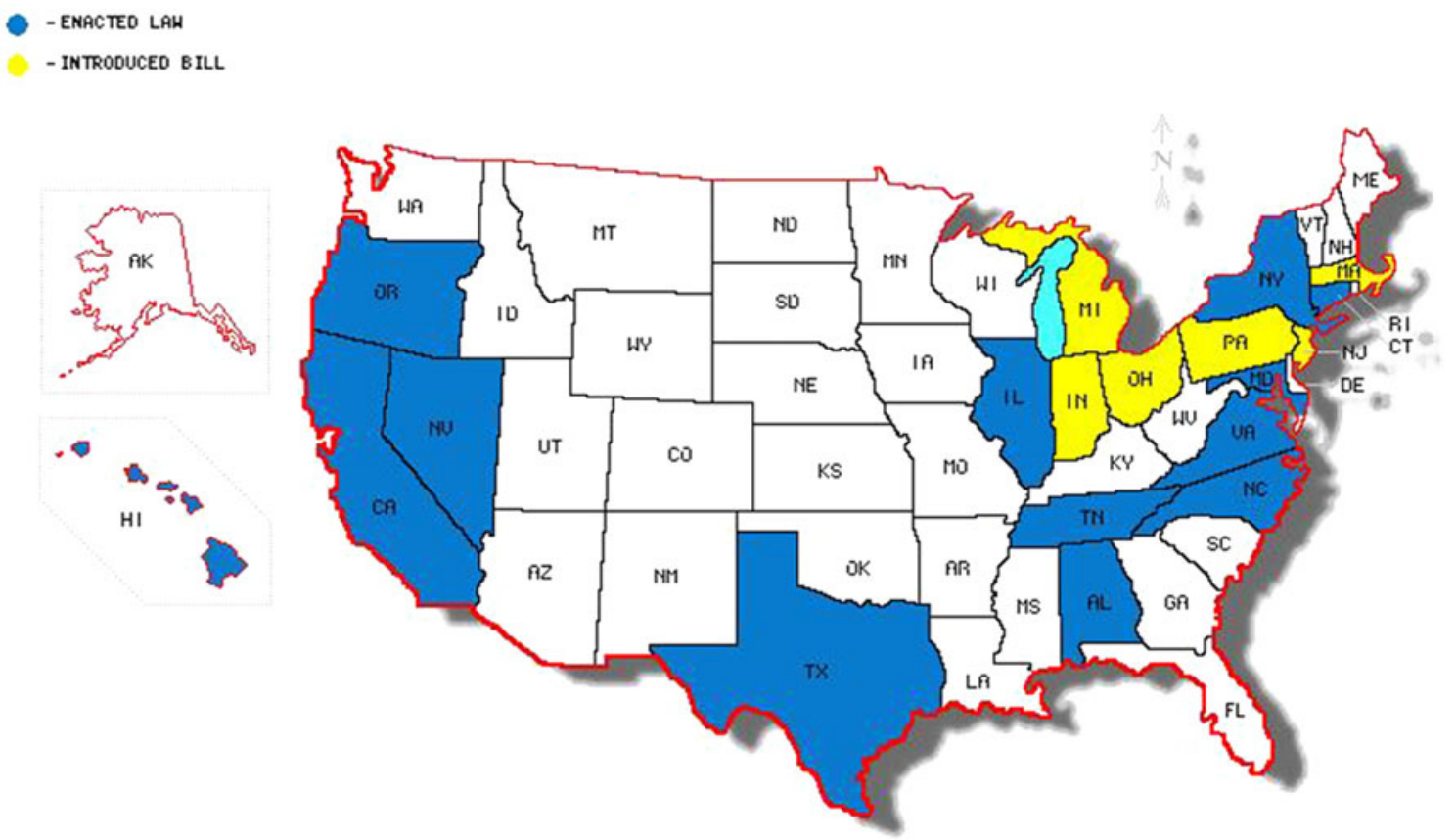

Fig. 2 Direct patient notification of breast density
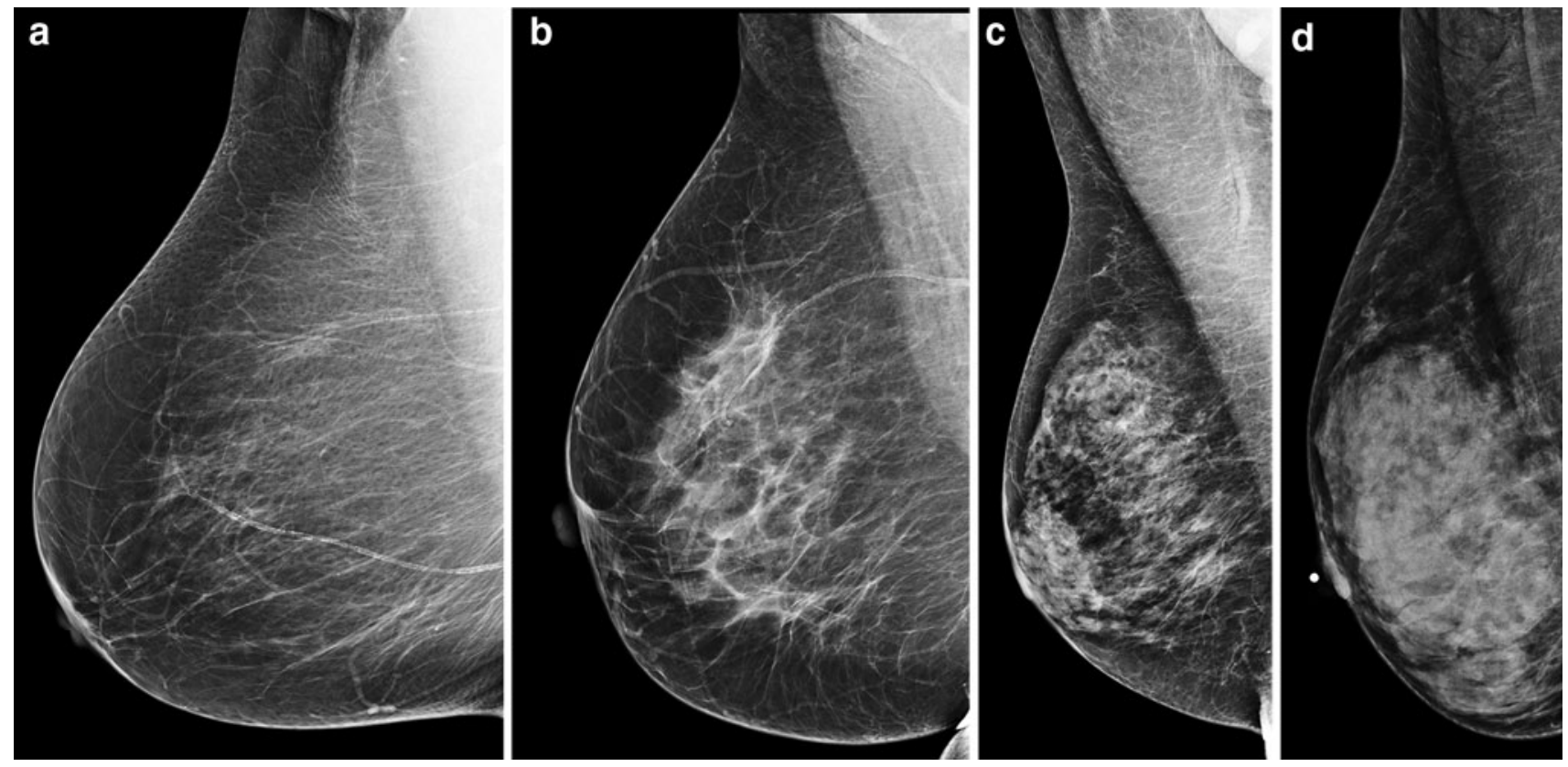

Fig. 3 BI-RADS density categories. a The breasts are almost entirely fatty; $\mathbf{b}$ there are scattered areas of fibroglandular density; $\mathbf{c}$ the breasts are heterogeneously dense, which may obscure detection of

notification legislation, the screening exams were technologist-performed, and they reported incremental cancer detection rates of about 2-3/1,000 [42•, 43, 44].

Despite these results, there is controversy as to whether screening ultrasound is a valid screening test. Major small masses; $\mathbf{d}$ the breasts are extremely dense, which lowers the sensitivity of mammography

disadvantages are the relatively large amount of time required to perform the examination, which puts a strain on personnel resources, the high false-positive rate and the very low positive predictive value (PPV) of biopsy recommendations. The PPV for ultrasound detected findings is 
$\sim 10 \%$ or less. In the large American College of Radiology Imaging Network 6666 study, the PPV was $8 \%$, and for the three Connecticut studies, it was 5-6 \% [37, 41-43]. In addition, in the study by Hooley et al. [42•], the BIRADS 3 rate where short interval follow-up was recommended was $20 \%$, meaning that one in five women was recommended to have a 6-month follow-up ultrasound examination for a probably benign finding, further adding to the drain on resources. In addition, there is not yet evidence that the use of screening ultrasound results in mortality reduction. It is clear, however, that with the increased awareness of breast density and its contribution to decreased sensitivity on mammography, more screening ultrasound examinations will be performed in this country even without evidence of mortality reduction.

\section{$M R I$}

In 2007, the ACS issued guidelines for the use of MRI as a screening tool in addition to mammography [45]. Based on the published literature and expert opinion, women at high risk for breast cancer, including those with a $20 \%$ or greater lifetime risk of breast cancer, BRCA mutation carriers and their untested first degree relatives as well as women with a past history of chest irradiation received between the ages of 10 and 30 were recommended to have annual MRI screening in addition to mammography. MRI has been shown to have higher sensitivity for breast cancer than mammography in these populations [46-48].

Because of the high level of risk in these women and the demonstrated poor performance of mammography in BRCA mutation carriers, there is little controversy about the use of MR despite the fact that there is no proven reduction in mortality associated with its use. What is more controversial is whether MR should be used in women with a moderate lifetime risk of $15-20 \%$ for whom the ACS guidelines state there was not enough evidence to advise either for or against the use of screening MRI. This group includes women with biopsy-proven lobular carcinoma in situ (LCIS), those with a personal history of breast cancer and women with extremely dense breasts. There are no data on the use of MRI for women whose only risk factor is dense breasts. There is, however, information that has been published after the ACS issued their guidelines in 2007 suggesting that MRI screening may be useful in women with a personal history of breast cancer and women with prior biopsy-proven LCIS. Two studies on screening MRI in women with a past history of LCIS showed similar supplemental cancer yields of 3.7 and $4.4 \%$, respectively $[49,50]$. A study of screening MR in women with a personal history of MR showed a cancer yield of $12 \%$ [51].

The downsides of screening with MR are high cost, need for intravenous contrast administration, variable insurance coverage and the fact that not all women are candidates for the examination because of claustrophobia, the presence of pacemakers or other metallic objects in the body, and renal impairment that precludes the use of contrast. Despite this and the fact that it too has not been shown to reduce breast cancer mortality, MRI is becoming increasingly utilized for supplemental screening of very high risk women.

\section{Molecular Breast Imaging}

With the development of gamma cameras with a limited field of view designed specifically for breast imaging, the use of nuclear medicine techniques using 99mtechnicium sestamibi for screening became a possibility. Rhodes et al. [52] at the Mayo Clinic studied 936 women with dense breasts and additional risk factors with both mammography and molecular breast imaging (MBI) using technicium $99 \mathrm{~m}$ sestamibi dedicated breast imaging. A total of 11 cancers were detected, 1 with mammography alone, 8 with sestimibi alone and 2 with both. These results are promising, but MBI at the currently used standard dose of $25 \mathrm{mCi}$ technicium $99 \mathrm{~m}$ sestamibi delivers a relatively high radiation dose to the breast as well as a high total body dose. The risk of radiation-induced cancer death is 20 times higher with MBI than for mammography at age 40 [53]. Therefore, it is difficult to justify the use of MBI at the current doses. Work is being done to reduce the dose while maintaining sensitivity, but until this is achieved, there is reluctance to use this technique for screening.

\section{Conclusion}

Despite its use for nearly 30 years in the US, screening mammography remains controversial, and the debate between advocates and opponents of screening is becoming increasingly acrimonious. Depending on the data used, the underlying assumptions and method of analysis, different conclusions as to the harms versus the benefits of screening mammography have been reached. What is not debated, however, is that mortality from breast cancer which had been increasing steadily in the years before screening was introduced, has decreased by $\sim 30 \%$ since its introduction [54]. It is very difficult to believe that screening has not had a role in this dramatic reduction and would be extremely unfortunate if the controversy surrounding screening mammography results in decreased utilization and a reversal of the gains achieved in morality reduction. Screening mammography has become integrated into routine health care for millions of American women and is unlikely to be abandoned, despite continuing controversy.

At the same time that screening with mammography is being challenged and recommendations for less rather than 
more screening are being issued, there is increased demand for supplemental screening with alternative imaging modalities including ultrasound and MRI. The role of these tests in screening and the effect, if any, on breast cancer mortality and health care costs remain to be established.

\section{Compliance with Ethics Guidelines}

Conflict of Interest Carol H. Lee declares that she has no conflicts of interest.

Human and Animal Rights and Informed Consent This article does not contain any studies with human or animal subjects performed by the author.

\section{References}

Papers of particular interest, published recently, have been highlighted as:

- Of importance

- Of major importance

1. Shapiro S, Venet W, Strax P, et al. 10- to 14-year effect of screening on breast cancer mortality. J Natl Cancer Inst. 1982;69:349-55.

2. Nystrom L, Rutqvist LE, Wall S, et al. Breast cancer screening with mammography: overview of Swedish randomised trials. Lancet. 1993;341:973-8.

3. Roberts MM, Alexander FE, Anderson TJ, et al. Edinburgh trial of screening for breast cancer: mortality at seven years. Lancet. 1990;335:241-6.

4. Hackshaw A. EUSOMA review of screening mammography. Ann Oncol. 2003;14:1193-5.

5. Nelson HD, Tyne K, Naik A, Bougatsos C, Chan BK, Humphrey L. Screening for breast cancer: an update for the US Preventive Services Task Force. Ann Int Med. 2009;151:1727-37.

6. Gotzsche PC, Olsen O. Is screening for breast cancer with mammography justifiable? Lancet. 2000;355:129-34.

7. Jorgensen KJ, Zahl P-H, Gotzsche PC. Breast cancer mortality in organized mammography screening in Denmark: comparative study. BMJ. 2010;320:c1241.

8. Autier P, Koechlin A, Smans M, Vatten L, Boniol M. Mammography screening and breast cancer mortality in Sweden. J Natl Cancer Inst. 2012;104:1080-93.

9. Kalger M, Zelen M, Langmark F, Adami H-O. Effect of screening mammography on breast-cancer mortality in Norway. N Engl J Med. 2010;363:1203-10.

10. •• Independent UK panel on breast cancer screening. The benefits and harms of breast cancer screening: an independent review. Lancet. 2012;380:1778-1786. This is a recent review of the evidence concerning screening mammography conducted by an independent body presumably without bias either for or against screening.

11. Tabar L, Vitak B, Chen TH-H, et al. Swedish two-county trial: impact of mammographic screening on breast cancer mortality during 3 decades. Radiology. 2011;260:658-63.

12. Nickson C, Mason KE, English DR, Kavanagh AM. Mammographic screening and breast cancer mortality: a case-control study and meta-analysis. Cancer Epidemiol Biomarkers Prev. 2012;21:1479-88.
13. - Webb ML, Cady B, Michaelson JS, Bush DM, Calvillo KZ, Kopans DB, Smith BL. A failure analysis of invasive breast cancer. Cancer. 2013. doi:10.1002/cncr.28199. This study showed that most breast cancer deaths occurred in women who had not had regular screening mammography.

14. US Preventive Services Task Force. Screening for breast cancer: US Preventive Services Task Force recommendation statement. Ann Int Med. 2009;151:716-26.

15. Miller $\mathrm{AB}$, Baines $\mathrm{CJ}$, To $\mathrm{T}$, et al. Canadian national breast screening study 1: breast cancer detection and death rates among women aged 40 to 49 years. Can Med Assoc J. 1992;147:1459-76.

16. Moss SM, Cuckle H, Evans A, Johns L, Waller M, Bobrow L. Effect of mammographic screening from age 40 years on breast cancer mortality at 10 years' follow-up: a randomised controlled trial. Lancet. 2006;368:2053-60.

17. Mandelblatt JS, Cronin KA, Bailey S, et al. Effects of mammography screening under different screening schedules: model estimates of potential benefits and harms. Ann Int Med. 2009; 151:738-47.

18. Kerlikowske K, Grady D, Barclay J, Sickles EA, Ernster V. Effect of age, breast density, and family history on the sensitivity of first screening mammography. JAMA. 1996;276:33-8.

19. Rosenberg RD, Yankaskas BC, Abraham LA, et al. Performance benchmarks for screening mammography. Radiology. 2006;241: $55-66$.

20. Christiansen CL, Wang F, Barton MB, Kreuter W, Elmore JG, Gelfand AE, Fletcher SW. Predicting the cumulative risk of falsepositive mammograms. J Natl Cancer Inst. 2000;92:1657-66.

21. Brewer NT, Salz T, Lille SE. The long-term effects of falsepositive mammograms. Ann Int Med. 2007;146:502-10.

22. Schwartz LM, Woloshin S, Sox HC, Fishchhoff B, Welch HG. US women's attitudes to false positive mammography and detection of ductal carcinoma in situ: cross sectional survey. BMJ. 2000;320:1635-40.

23. Jorgensen KJ, Gotzsche PC. Overdiagnosis in publicly organised mammography screening programmes: systematic review of incidence trends. BMJ. 2009;329:b2587.

24. Yen AM-F, Duffy SW, Chen Tony H-H, et al. Long-term incidence of breast cancer by trial arm in one county of the Swedish two-county trial of mammographic screening. Cancer. 2012;118: 5728-32.

25. - Bleyer A, Welch HG. Effect of three decades of screening mammography on breast-cancer incidence. N Engl J Med. 2012;367:1998-2005. This paper presents one of the strongest arguments against screening mammography, namely that of overdiagnosis.

26. Hofvind S, Lee CI, Elmore JG. Stage-specific breast cancer incidence rates among participants and non-participants of a population-based breast cancer screening program. Breast Cancer Res Treat. 2012;135:291-9.

27. Poplack SP, Tosteson TD, Cogel CA, Nagy HM. Digital breast tomosynthesis: initial experience in 98 women with abnormal digital screening mammography. AJR Am J Roentgenol. 2007; 189:616-23.

28. Michell MJ, Iqbal A, Wasan RK, et al. A comparison of the accuracy of film-screen mammography, full-field digital mammography, and digital breast tomosynthesis. Clin Radiol. 2012; 67:976-81.

29. Rafferty EA, Park JM, Philpotts LE, Poplack SP, Sumkin JH, Halpern EF, Niklason LT. Assessing radiologist performance using combined digital mammography and breast tomosynthesis compared with digital mammography alone: results of a multicenter, multireader trial. Radiology. 2013. doi:10.1148/radiol. 12120674.

30. - Skaane P, Gullien R, Bjorndal H, et al. Digital breast tomosynthesis (DBT): initial experience in a clinical setting. Acta 
Radiol. 2012;53:524-529. This is the first report of a large, ongoing prospective trial of this emerging technology.

31. Ciatto S, Houssami N, Bernardi D, et al. Integration of 3D digital mammography with tomosynthesis for population breast-cancer screening (STORM): a prospective comparison study. Lancet Oncol. 2013;14:583-9.

32. Rose SL, Tidwell AL, Bujnoch LJ, Kuswaha AC, Nordmann AS, Sexton R. Implementation of breast tomosynthesis in a routine screening practice: an observational study. AJR Am J Roentgenol. 2013;200:1401-8.

33. Haas BM, Kaira V, Geisel J, Raghu M, Durand M, Philpotts LE. Comparison of tomosynthesis plus digital mammography and digital mammography alone for breast cancer screening. Radiology. 2013. doi:10.1148/radiol.13130307.

34. Kaplan SS. Clinical utility of bilateral whole-breast US in the evaluation of women with dense breast tissue. Radiology. 2001;221:641-9.

35. Kolb TM, Lichy J, Newhouse JH. Comparison of the performance of screening mammography, physical examination, and US and evaluation of factors that influence them: an analysis of 27,825 patient evaluations. Radiology. 2002;225:165-75.

36. Crystal P, Strano SD, Shcharynski S, Koretz MJ. Using sonography to screen women with mammographically dense breasts. AJR Am J Roentgenol. 2003;181:177-82.

37. Berg WA, Blume JD, Cormack JB, et al. Combined screening with ultrasound and mammography vs mammography alone in women at elevated risk of breast cancer. JAMA. 2008;299: 2151-63.

38. •• www.areyoudense.org. Accessed 17 Oct 2013. This website represents a nationwide effort that has resulted in legislation mandating direct patient notification of mammographic breast density. This is resulting in increased utilization of screening ultrasound.

39. Breast Imaging Reporting and Data System ${ }^{\circledR}$. 4th ed. Reston: American College of Radiology; 2003.

40. Pisano ED, Gatsonis C, Hendrick E, et al. Diagnostic performance of digital versus film mammography for breast-cancer screening. N Engl J Med. 2005;353:1773-83.

41. Carney PA, Miglioretti DL, Yankaskas BC, et al. Individual and combined effects of age, breast density, and hormone replacement therapy use on the accuracy of screening mammography. Ann Int Med. 2003;138:168-75.

42. • Hooley RJ, Greenberg KL, Stackhouse RM, Geisel JL, Butler RS, Philpotts LE. Screening US in women with mammographically dense breasts: initial experience with Connecticut public act 09-41.
Radiology. 2012;265:59-69. This is a report of the performance of technologist performed screening ultrasound in actual clinical setting describing the outcome and impact of direct patient notification laws.

43. Weigert J, Steenbergen S. The Connecticut experiment: the role of ultrasound in the screening of women with dense breasts. Breast J. 2012;18:517-22.

44. Parris T, Wakefield D, Frimmer H. Real world performance of screening breast ultrasound following enactment of Connecticut bill 458. Breast J. 2013;19:64-70.

45. Saslow D, Boetes C, Burke W, et al. American Cancer Society guidelines for breast screening with MRI as an adjunct to mammography. CA Cancer J Clin. 2007;57:75-89.

46. Kriege M, Brekelmans CT, Boetes C, et al. Efficacy of MRI and mammography for breast cancer screening in women with a familial or genetic predisposition. N Engl J Med. 2004;351: 427-37.

47. Leach MO, Boggis CR, Dixon AK, et al. Screening with magnetic resonance imaging and mammography of a UK population at high familial risk of breast cancer: a prospective multicentre cohort study (MARIBS). Lancet. 2005;365:1769-78.

48. Warner E, Messersmith H, Causer P, et al. Systematic review: using magnetic resonance imaging to screen women at high risk for breast cancer. Ann Intern Med. 2008;148:671-9.

49. Sung JS, Malak SF, Bajaj P, Alis R, Dershaw DD, Morris EA. Screening breast MR imaging in women with a history of lobular carcinoma in situ. Radiology. 2011;261:414-20.

50. Friedlander LC, Roth SO, Gavenonis SC. Results of MR imaging screening for breast cancer in high-risk patients with lobular carcinoma in situ. Radiology. 2011;26:421-7.

51. Brennan S, Liberman L, Dershaw DD, Morris E. Breast MRI screening of women with a personal history of breast cancer. AJR Am J Roentgenol. 2010;195:510-6.

52. Rhodes DJ, Hruska CB, Phillips SW, Whaley DH, O'Connor MK. Dedicated dual-head gamma imaging for breast cancer screening in women with mammographically dense breasts. Radiology. 2011;258:106-18.

53. O'Connor MK, Rhodes DJ, Hruska CB, Clancy CB, Vetter RJ. Comparison of radiation exposure and associated radiationinduced cancer risks from mammography and molecular imaging of the breast. Med Phys. 2010;37:6187-98.

54. http://seer.cancer.gov/statfacts/html/breast.html\#incidence-mortality. Accessed 17 Oct 2013. 